\title{
Retrospective Correction of MR Intensity Inhomogeneity by Information Minimization
}

\author{
Boštjan Likar ${ }^{1}$, Max A. Viergever ${ }^{2}$, and Franjo Pernuš ${ }^{1}$ \\ ${ }^{1}$ University of Ljubljana, Department of Electrical Engineering \\ Tržaška 25, 1000 Ljubljana, Slovenia \\ bostjan.likar@fe.uni-lj.si \\ franjo.pernus@fe.uni-lj.si \\ 2 Image Sciences Institute, University Hospital Utrecht \\ P.O. Box 85500, 3508 GA Utrecht, The Netherlands \\ max@isi.uu.nl
}

\begin{abstract}
In this paper the problem of retrospective correction of intensity inhomogeneity in MR images is addressed. A novel model-based correction method is proposed, based on the assumption that an image corrupted by intensity inhomogeneity contains more information than the corresponding uncorrupted image. The image degradation process is described by a linear model, consisting of a multiplicative and an additive component which are modeled by a combination of smoothly varying basis functions. The degraded image is corrected by the inverse of the image degradation model. The parameters of this model are optimized such that the information of the corrected image is minimized while the global intensity statistic is preserved. The method was quantitatively evaluated and compared to other methods on a number of simulated and real MR images and proved to be effective, reliable, and computationally attractive.
\end{abstract}

\section{Introduction}

In magnetic resonance imaging (MRI), image intensity inhomogeneity is an adverse phenomenon which manifests itself by slow intensity variations of the same tissue class over the image domain. Intensity inhomogeneity may be caused by a number of factors including poor radio frequency (RF) coil uniformity, static field inhomogeneity, RF penetration, gradient-driven eddy currents, and overall patient anatomy and position [1], [2]. Spurious intensity variations, which may reach up to $30 \%$ of the image intensity amplitude [3], [4] usually do not affect the visual impression of the image significantly, but may have serious implications for MR image analysis, e.g., in segmentation, registration, or quantification.

Methods for correction of intensity inhomogeneities may be prospective or retrospective. The former require an acquisition protocol tuned to inhomogeneity correction, while the latter can be applied to any MR image, since they only use the information naturally occurring in an image. A number of prospective methods have been introduced in MRI, which either use phantom acquisitions [1], [2], [5]-[8] or measure the excitation field [9] or the static uniformity of the reception coil [10] in vivo. However, the patient-independence requirements and/or extended scan time 
makes them impractical for clinical use. As an alternative, a number of retrospective methods have been proposed. The most intuitive retrospective methods for correcting multiplicative and smooth intensity variations are homomorphic filtering (see e.g. [11]), image blurring [7], smoothing [13], averaging [14], Fourier domain filtering [15], and homomorphic unsharp masking [16]. The histogram matching method was also applied [17]. A number of methods were proposed that fit polynomials or thinplate splines to manually or automatically selected points [12], to regions defined by inhomogeneity-tolerant preliminary segmentation [3], or directly to image data by using multiple-valley criterion functions instead of least squares [18]. Sled et al. presented an iterative optimization method for MRI, which seeks the smooth multiplicative field that maximizes the frequency content of the distribution of tissue intensity [19]. A segmentation-based method, using the expectation-minimization algorithm to iteratively classify and correct the image, was introduced in [20], improved in [4], and recently further extended in [21]. Segmentation-based methods that use fuzzy C-means clustering can be found in [22]-[24], while the usage of Markov random fields was considered in [25]. Filtering out and weighted reintegration of well-characterized local derivatives to estimate the bias field was proposed by Vokurka et al. [26]. In similar vein, in [27] a new variational shapefrom-orientation approach was suggested. A review and evaluation of MRI nonuniformity corrections for brain tumor response measurements was provided by Velthuizen et al. [28], reporting that different methods give significantly different correction images and thus concluding that non-uniformity correction is not yet well understood.

In a recent paper [29], we described a retrospective shading correction method based on entropy minimization, designed for correcting the intensity inhomogeneity in 2D images. In this paper, we generalize the method to 3D MR images. The derivation of our algorithm is based on the assumption that an image corrupted by intensity inhomogeneity contains more information than the corresponding uncorrupted image. The method will be quantitatively evaluated and compared with two recently proposed methods, [19] and [26], on a large number of simulated and real MR images.

\section{Theory}

\subsection{Problem Formulation}

Let $v(x)$ denote the acquired image and let $u(x)$ denote the "true" image of the imaged object. The two images are related by:

$$
v(x)=f(u(x)) \text { or } u(x)=f^{-1}(v(x))
$$

with $f$ denoting the image degradation model (bias field) that introduces a spatially dependent intensity degradation to the true image $u(x)$ and $f^{-1}$ representing the inverse of the degradation model. The problem of retrospective correction of intensity inhomogeneity is to find the true image $u(x)$ from the acquired image $v(x)$. 


\subsection{Correction Strategy}

The proposed correction strategy is based on the assumption that because of the image degradation process the information content of the acquired image will be higher that the information content of the true image:

$$
I[v(x)]=I[f(u(x))]>I[u(x)]
$$

The information $I$ of any image $v(x)$ can be quantitatively expressed by the Shannon-Wiener entropy $H[v(x)]$ as:

$$
I[v(x)]=H[v(x)]=-\sum_{n} p(n) \log p(n)
$$

where $p(n)$ is the probability that a point in image $v(x)$ has value $n$.

Suppose a correction model $\widetilde{f}^{-1}$, which is constrained in such a way that it can not change the mean intensity of the input image $v(x)$ and can not transform the input image to a uniform image, is given. Then, an estimate $\widetilde{u}(x)$ may be obtained as:

$$
\widetilde{u}(x)=\widetilde{f}^{-1}(v(x))
$$

According to the assumption made in (2), transforming the image $v(x)$ by the correction model $\widetilde{f}^{-1}(4)$ in such a way that the information $I$ of the transformed image $\widetilde{u}(x)$ is minimized should lead to the optimal correction model $\widetilde{f}_{o}^{-1}$ :

$$
\widetilde{f}_{o}^{-1}=\arg \underset{\widetilde{f}^{-1}}{\min }\left\{I\left[\widetilde{f}^{-1}(v(x))\right]\right\}
$$

which defines the transformation of the image $v(x)$ to the corrected image $\widetilde{u}_{o}(x)$ :

$$
\widetilde{u}_{o}(x)=\widetilde{f}_{o}^{-1}(v(x))
$$

\section{Correction by a Linear Model}

\subsection{Modeling}

The linear model of image degradation consists of a multiplicative $m(x)$ and an additive $a(x)$ intensity degradation component:

$$
v(x)=u(x) m(x)+a(x)
$$

By the inverse of the degradation model, the estimation $\widetilde{u}(x)$ is obtained as:

$$
\widetilde{u}(x)=v(x) \widetilde{m}^{-1}(x)+\widetilde{a}^{-1}(x)
$$

where

$$
\widetilde{m}^{-1}(x)=\frac{1}{\widetilde{m}(x)} \quad \text { and } \quad \widetilde{a}^{-1}(x)=-\frac{\widetilde{a}(x)}{\widetilde{m}(x)}
$$


are the multiplicative and additive correction components, respectively. These two components are described by smoothly varying basis functions $s_{i}(x)$ :

$$
\widetilde{m}^{-1}(x)=\sum_{i=1}^{K}{ }^{m} b_{i}{ }^{m} S_{i}(x) \quad \text { and } \quad \widetilde{a}^{-1}(x)=\sum_{i=1}^{K}{ }^{a} b_{i}{ }^{a} S_{i}(x)
$$

that are uniquely defined by parameters $m b$ and $a b$, respectively.

To neutralize the global transformation effect (8) the correction components have on the acquired image $v(x)$, we introduce the mean-preserving condition:

$$
\frac{1}{\Theta} \int_{\Omega} v(x) d \Omega=\frac{1}{\Theta} \int_{\Omega}\left(v(x) \widetilde{m}^{-1}(x)+\widetilde{a}^{-1}(x)\right) d \Omega
$$

which ensures that the mean intensity values of $v(x)$ and $\widetilde{u}(x)$, defined over the relevant image domain $\Omega$ of size $\Theta ; \Theta=\int_{\Omega} d \Omega$, will be the same.

To equalize the mean absolute contributions of individual basis functions $s_{i}(x)$ to the global intensity transformation, the parameters ${ }^{m} b$ and $a b$ are normalized:

$$
\frac{1}{\Theta} \int_{\Omega}\left|v(x)^{m} S_{i}(x)\right| d \Omega=1 \quad \text { and } \quad \frac{1}{\Theta} \int_{\Omega}\left|{ }^{a} S_{i}(x)\right| d \Omega=1 \quad \text { for all } i
$$

As the smoothly varying basis functions $s_{i}(x)$ we use polynomial terms $q_{i}(x)$ in the following form:

$$
{ }^{m} S_{i}(x)=\frac{q_{i}(x)-{ }^{m} c_{i}}{{ }^{m} d_{i}} \quad \text { and } \quad{ }^{a} S_{i}(x)=\frac{q_{i}(x)-{ }^{a} c_{i}}{{ }^{a} d_{i}}
$$

with neutralization constants $c_{i}$, needed to fulfil the mean-preserving condition (11), and normalization constants $d_{i}$, needed to fulfil (12). The two correction components $\widetilde{m}^{-1}(x)$ and $\widetilde{a}^{-1}(x)$, which define the correction model (8), are modeled by the linear combination of neutralized and normalized polynomials that fulfil (11) and (12):

$$
\widetilde{m}^{-1}(x)=1+\sum_{i=2}^{K}{ }^{m} b_{i} \frac{q_{i}(x)-{ }^{m} c_{i}}{{ }^{m} d_{i}} \quad \text { and } \quad \widetilde{a}^{-1}(x)=\sum_{i=2}^{K} a^{a} b_{i} \frac{q_{i}(x)-{ }^{a} c_{i}}{{ }^{a} d_{i}}
$$

\subsection{Correction}

The optimal parameters $m b_{o}$ and $a b_{o}$ are found by Powell's multi-dimensional directional set method and Brent's one-dimensional optimization algorithm [30]:

$$
\left\{{ }^{m} b_{o},{ }^{a} b_{o}\right\}=\arg \min _{\{m b, a b}\left\{I\left[v(x) \widetilde{m}^{-1}(x)+\widetilde{a}^{-1}(x)\right]\right\}
$$

The optimal parameters ${ }^{m} b_{o}$ and $a b_{o}$ define the optimal components $\widetilde{m}_{o}^{-1}(x)$ and $\widetilde{a}_{o}^{-1}(x)$, respectively, which transform the acquired image $v(x)$ into the optimally corrected image $\widetilde{u}_{o}(x)$ :

$$
\widetilde{u}_{o}(x)=v(x) \widetilde{m}_{o}^{-1}(x)+\widetilde{a}_{o}^{-1}(x)
$$




\subsection{Implementation Details}

Three variations of the proposed information minimization method are implemented. In the first method, named MA2, the multiplicative and additive component are modeled by a second order polynomial. The other two methods, named M2 and M4, consist solely of a multiplicative component, which is modeled by a second and fourth order polynomial, respectively.

The correction domain $\Omega$ is extracted by thresholding the image and subsequently eroding the obtained non-background area, using a simple six-voxel structuring element. Prior to the optimization process, the neutralization constants $c_{i}$ and normalization constants $d_{i}$ of the correction components (14) are calculated.

Effective calculation of the entropy $H$ is crucial for the performance of the proposed method. The entropy (3) is defined from a set of probabilities $p(n)$ that can be obtained from the intensity histogram of the current estimate $\widetilde{u}(x)$. Since $\widetilde{u}(x)$ is obtained by an intensity transformation applied to a given image, an integer gray value $g$ is transformed to a new real value $g^{\prime}$, which in general lies between two integer values, say $k$ and $k+1$. We use partial intensity interpolation by which the histogram entries $h(k)$ and $h(k+1)$ are fractionally updated by $k+1-g^{\prime}$ and $g^{\prime}-k$, respectively. Prior to the calculation of the set of probabilities $p(n)$, the histogram $h(n)$ is slightly blurred to reduce the effects of imperfect intensity interpolation. To provide a high statistical power, which is proportional to the number of samples used to form a $1 \mathrm{D}$ histogram from a 3D image, we use 8 bit intensity quantization. Due to the high statistical power, histograms may be formed by substantially sub-sampling the image data without affecting the method's performance.

\section{Experiments and Results}

\subsection{Validating the Correction Strategy}

In this section we tested the proposed correction strategy using the MA2 method. For this purpose, two simulated MR T1-weighted images [31]-[33], one with $0 \%$ (uniform image) and the other with $40 \%$ intensity non-uniformity, were used. In Fig. 1 the recovery of the intensity distribution can be observed by comparing the histograms of the uniform, non-uniform, and corrected image.

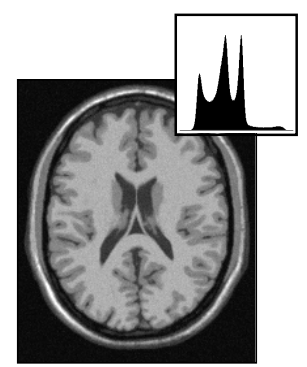

Uniform

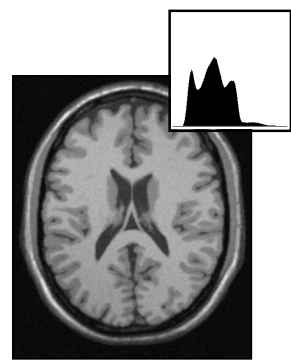

Non-uniform

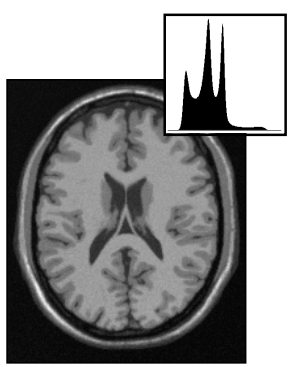

Corrected

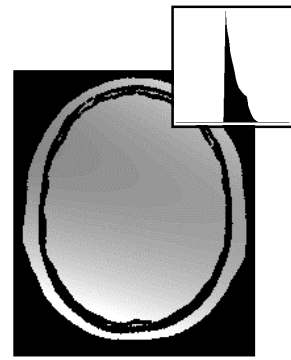

Correction field

Fig. 1. Simulated uniform and non-uniform (40\% intensity inhomogeneity) images, and the corresponding retrospective correction of the non-uniform image. 


\subsection{Evaluation}

Methods. Five methods were included in the quantitative evaluation, i.e. the so-called MA2, M2, and M4 variations of the information minimization method described in this paper, the nonparametric nonuniform intensity normalization (N3) method proposed by Sled et al. [19], and the fast model independent (FMI) method introduced by Vokurka et al. [26]. The N3 and FMI methods were implemented at our site by using the code available via internet at [34] and [35], respectively. The implementations were validated. The parameters of the N3 and FMI methods were set to their default values.

Experimental data. One set of simulated and four sets of real MR data were used:

Set 1: Consisted of $12(4 \times \mathrm{T} 1, \mathrm{~T} 2$, and PD-weighted) MRI volumes from the BrainWeb MR simulator $(181 \times 217 \times 181$ voxels, 8 bit, $1 \mathrm{~mm}$ slice thickness, $3 \%$ noise) [31]-[33].

Set 2: Six ( $2 \times \mathrm{T} 1, \mathrm{~T} 2$, and PD-weighted) MRI volumes, 3 of a normal volunteer (256x $256 \times 25$ voxels, 8bit) and 3 of a brain tumor patient with multiple malignant meningioma lesions $(256 \times 256 \times 20$ voxels, 8 bit $)$, were used. A complete gray and white matter manual segmentation was available for each volume [36].

Set 3: The set contained 33 (11 x T1, T2, and PD-weighted $)$ real MR images $(181 \times 217 \times 181$ voxels, 8 bit) with manual labeling of the regions of pure gray and white matter, acquired at 11 different sites from 11 multiple sclerosis patients having a moderate number of white matter lesions [19].

Set 4: The set contained 18 normal coronal three-dimensional T1-weighted spoiled gradient echo MRI scans acquired on two different imaging systems. A complete segmentation was available [37]-[39]. The images were all 8 bit with the resolution of either $128 \times 128$ or $160 \times 160$ and they had 50 to 58 slices.

Set 5: A breast T1-sagittal image (1T Siemens Magnetom Harmony, body coil, $4 \mathrm{~mm}$ slice thickness, $256 \times 256 \times 20$ voxels, 8 bit) and a prostate T1-axial image (1T Siemens Magnetom Harmony, phased array, $3 \mathrm{~mm}$ slice thickness, $256 \times 256 \times 20$ voxels, 8 bit) were used.

Criteria. The performance of an intensity non-uniformity correction method is commonly evaluated by comparing the coefficients of variations $(c v)$ within the individual tissue classes in the original and corrected images. The $c v$, which is invariant to the uniform multiplicative intensity transformation, is computed as:

$$
c v(C)=\frac{\sigma(C)}{\mu(C)}
$$

where $\sigma(C)$ and $\mu(C)$ are the standard deviation and mean intensity of class $C$, respectively. A drawback is that $c v$ does not provide any information on the overlap between the intensity distributions of distinct tissue classes. To estimate the overlap we introduce the so-called coefficient of joint variations $\operatorname{cjv}\left(C_{l}, C_{2}\right)$ : 


$$
\operatorname{cjv}\left(C_{1}, C_{2}\right)=\frac{\sigma\left(C_{1}\right)+\sigma\left(C_{2}\right)}{\left|\mu\left(C_{1}\right)-\mu\left(C_{2}\right)\right|}
$$

which is the sum of the standard deviations of two distinct classes; $C_{1}$ and $C_{2}$, normalized by the difference of means. The $c j v$ is invariant to the uniform linear, i.e. multiplicative and additive, intensity transformation and is thus a more general quantitative measure than the $c v$.

Results. Quantitative evaluation of the MA2, M2, M4, N3, and FMI methods was performed by computing the $c j v(\mathrm{GM}, \mathrm{WM})$ of the gray and white matters for all images from the first four experimental sets.

Table 1 contains the results of non-uniformity correction of the images from the first two sets. Ideally the $c j v$ of the simulated uniform and corrected uniform images of set 1 should be identical. This was approximately true for all methods except the FMI method, which corrupted the images with $0 \%$ non-uniformity. The corrected images with simulated $40 \%$ intensity non-uniformity indicate that all methods, except the FMI, can efficiently reduce the overlap of their intensity distributions. The $c j v$-s of all corrected images with $40 \%$ non-uniformity are similar to the $c j v$-s of the images with $0 \%$ non-uniformity for all methods, except for the FMI method and the N3 method when applied to a normal T2 image. The methods applied to the real normal and tumor volumes (set 2) yielded similar reductions of the non-uniformity criteria, with the exception of M4 method, which slightly increased the $c j v$ of T2 tumor volume, and the FMI method, which increased the $c j v$ of the three normal volumes.

Table 1. Correction of simulated and real data of image sets 1 and 2.

\begin{tabular}{ccc|cccccc}
\multicolumn{1}{c}{} & \multicolumn{10}{c}{$c j v(\mathrm{GM}, \mathrm{WM})$ in [\%] } \\
\hline Set & Mod & Bias & Orig. & MA2 & M2 & M4 & N3 & FMI \\
\hline 1 - Normal & T1 & $0 \%$ & 51.6 & 52.0 & 52.1 & 52.1 & 51.9 & 72.5 \\
(simulated) & & $40 \%$ & 69.3 & 51.6 & 51.5 & 51.7 & 51.8 & 96.4 \\
& T2 & $0 \%$ & 83.2 & 82.1 & 82.6 & 82.5 & 83.1 & 125.5 \\
& & $40 \%$ & 106.4 & 82.3 & 82.4 & 82.4 & 103.9 & 116.8 \\
& PD & $0 \%$ & 64.9 & 64.7 & 64.7 & 65.0 & 66.4 & 93.5 \\
& & $40 \%$ & 163.0 & 64.3 & 64.5 & 64.1 & 66.7 & 107.9 \\
\hline 1 - MS lesions & T1 & $0 \%$ & 50.9 & 51.4 & 51.5 & 51.5 & 51.3 & 88.0 \\
(simulated) & & $40 \%$ & 68.0 & 50.8 & 50.8 & 51.1 & 51.1 & 65.3 \\
& T2 & $0 \%$ & 74.9 & 74.1 & 74.2 & 74.3 & 74.9 & 150.3 \\
& & $40 \%$ & 123.8 & 73.7 & 74.1 & 74.2 & 77.8 & 98.3 \\
& PD & $0 \%$ & 66.9 & 66.7 & 66.7 & 67.0 & 68.7 & 117.3 \\
& & $40 \%$ & 195.6 & 66.5 & 66.6 & 66.1 & 68.7 & 125.9 \\
\hline 2 - Normal & T1 & & 138.0 & 131.4 & 133.0 & 134.0 & 127.0 & 144.3 \\
(real) & T2 & & 90.7 & 88.4 & 90.4 & 89.8 & 90.7 & 105.0 \\
& PD & & 77.8 & 70.7 & 72.1 & 72.1 & 73.2 & 87.1 \\
\hline 2 - Tumor & T1 & & 175.7 & 167.5 & 168.6 & 170.4 & 168.1 & 173.6 \\
(real) & T2 & & 169.3 & 164.7 & 166.6 & 175.5 & 161.3 & 163.8 \\
& PD & & 133.8 & 118.0 & 115.2 & 118.2 & 114.6 & 121.3 \\
\hline
\end{tabular}

Table 2 summarizes the mean changes of the $c j v(\mathrm{GM}, \mathrm{WM})$ for the image sets 3 and 4 . The FMI method performed poorly on nearly all images from the two sets. On T1 and T2 images from set 3 the $c j v$ were best reduced by the M4 method. The MA2 method 
was the most effective in correcting the PD images from set 3 , while the T1 images from set 4 were again best corrected by the M4 method.

Table 2. Mean changes of $c j v(\mathrm{GM}, \mathrm{WM})$ in [\%] for the images from sets 3 and 4.

\begin{tabular}{ccccccc} 
Set & Mod & MA2 & M2 & M4 & N3 & FMI \\
\hline 3 & T1 & -4.39 & -2.78 & -5.92 & -0.28 & 44.62 \\
3 & T2 & -18.10 & -23.65 & -29.58 & -21.69 & 17.47 \\
3 & PD & -35.76 & -27.97 & -30.66 & -28.90 & -2.19 \\
4 & T1 & -0.14 & -0.04 & -3.54 & -0.12 & 5.56 \\
\hline
\end{tabular}

In general, the M4 method performed the best from the methods tested and was also faster than the N3 and FMI methods. A successful application of the M4 method to the non-brain images from set 5 is illustrated in Fig. 2.

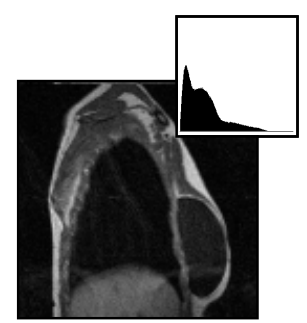

Original

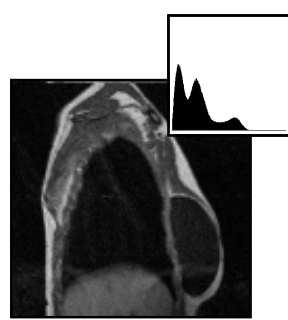

Corrected

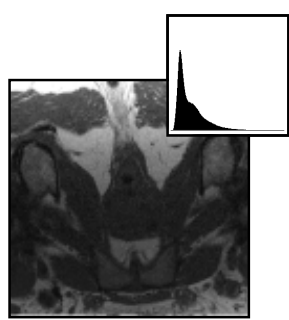

Original

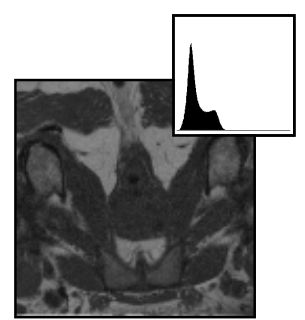

Corrected

Fig. 2. Correction of the breast and prostate image by the M4 method.

\section{Conclusion}

A novel information-theoretic approach to retrospective correction of intensity inhomogeneities in MRI, based on modeling the bias field and minimizing the information of the acquired images by the parametric polynomial model, was proposed. The modeling approach offers the possibility of incorporating knowledge about the image formation process in the correction algorithm, while the global intensity uniformity criterion, i.e. the entropy, enables the optimization of arbitrarily complex image formation models. The method can be widely applied to different types of MR images because it solely uses the information that is naturally present in an image, without making assumptions on its spatial and intensity distribution. The proposed method requires no preprocessing, parameter setting, nor user interaction and my thus be a valuable tool in MR image analysis.

\section{Acknowledgements}

This work was supported by the Ministry of Science and Technology of the Republic of Slovenia under grant J2-0659-1538 and by the IST-1999-12338 project, funded by the European Commission. The authors express their sincere thanks to Robert P. Velthuizen, John G. Sled, Elizabeth A. Vokurka, Chris Stork, and Andrew J. Worth for their generous help and cooperation. 


\section{References}

1. B. R. Condon, J. Patterson, D. Wyper, A. Jenkins, and D. M. Hadley, "Image nonuniformity in magnetic resonance imaging: its magnitude and methods for its correction." The British Journal of Radiology, 60:83-87, 1987.

2. A. Simmons, P. S. Tofts, G. J. Barker, and S. Arridge, "Sources of intensity nonuniformity in spin echo Images at 1.5 T." Magnetic Resonance in Medicine, 32:121-128, 1994.

3. C. R. Meyer, P. H. Bland, and J. Pipe, "Retrospective correction of intensity inhomogeneities in MRI." IEEE Transactions on Medical Imaging, 14:36-41, 1995.

4. R. Guillemaud and M. Brady, "Estimating bias field of MR images." IEEE Transactions on Medical Imaging, 16:238-251, 1997.

5. D. A. G. Wicks, G. J. Barker, and P. S. Tofts, "Correction of intensity nonuniformity in MR images of any orientation." Magnetic Resonance Imaging, 11:183-196, 1993.

6. M. Tincher, C. R. Meyer, R. Gupta, and D. M. Williams, "Polynomial modeling and reduction of RF body coil spatial inhomogeneity in MRI." IEEE Transactions on Medical Imaging, 12:361-365,1993.

7. L. Axel, J. Costantini, and J. Listerud, "Intensity correction in surface-coil MR imaging." American Journal of Radiology, 148:418-420, 1987.

8. E. R. McVeigh, M. J. Bronskill, and R.M. Henkelman, "Phase and sensitivity of receiver coils in magnetic resonance imaging." Medical Physics, 13:806-814, 1986.

9. R. Stollberger and P. Wach, "Imaging of the active $\mathrm{B}_{1}$ field in vivo." Magnetic Resonance in Medicine, 35:246-251, 1996.

10. P. A. Narayana, W. W. Brey, M. V. Kulkarni, and C. L. Sivenpiper, "Compensation for surface coil sensitivity variation in magnetic resonance imaging." Magnetic Resonance Imaging, 6:271-274, 1988 .

11. B. Johnson, M. S. Atkins, B. Mackiewich, and M. Anderson, "Segmentation of multiple sclerosis lesions in intensity corrected multispectral MRI." IEEE Transactions on Medical Imaging, 15:154-169, 1996.

12. B. M. Dawant, A. P. Zijdenbos, and R. A. Margolin, "Correction of intensity variations in MR images for computer-aided tissue classification." IEEE Transactions on Medical Imaging, 12:770-781, 1993

13. W. W. Brey and P. A. Narayana, "Correction for intensity falloff in surface coil magnetic resonance imaging." Medical Physics, 15:241-245, 1988.

14. A. Koivula, J. Alakuijala, and O. Tervonen, "Image feature based automatic correction of low-frequency spatial intensity variations in MR images." Magnetic Resonance Imaging, 15:1167-1175, 1997

15. J. Haselgrove and M. Prammer, "An algorithm for compensation of surface-coil images for sensitivity of the surface coil." Magnetic Resonance Imaging, 4:469-472, 1986.

16. B. H. Brinkmann, A. Manduca, and R. A. Robb, "Optimized homomorphic unsharp masking for MR greyscale inhomogeneity correction." IEEE Transactions on Medical Imaging, 17:161-171, 1998.

17. L. Wang, H. M. Lai, G. J. Barker, D. H. Miller, and P. S. Tofts, "Correction for variations in MRI scanner sensitivity in brain studies with histogram matching." Magnetic Resonance in Medicine, 39:322-327, 1998.

18. C. Brechbühler, G. Gerig, and G. Székely, "Compensation of spatial inhomogeneity in MRI based on a parametric bias estimate.", in Visualisation in Biomedical Computing, Proceedings of VBC'96, Lecture Notes in Computer Science, 1131:141-146, 1996, Springer-Verlag.

19. J. G. Sled, A. P. Zijdenbos, and A. C. Evans, "A nonparametric method for automatic correction of intensity nonuniformity in MRI data." IEEE Transactions on Medical Imaging, 17:87-97, 1998.

20. W. M. Wells III, W. E. L. Grimson, and F. A. Jolesz, "Adaptive segmentation of MRI data." IEEE Transactions on Medical Imaging, 15:429-442, 1996. 
21. K. van Leemput, F. Maes, D. Vandermeulen, and P. Suetens, “Automated model-based bias field correction of MR images of the brain." IEEE Transactions on Medical Imaging, 18:885-896, 1999.

22. S. K. Lee and M. W. Vannier, "Post-acquisition correction of MR inhomogeneity." Magnetic Resonance in Medicine, 36:275-286, 1996.

23. D. L. Pham and J. L. Prince, "An adaptive fuzzy C-means algorithm for image segmentation in the presence of intensity inhomogeneities." Pattern Recognition Letters, 20:57-68, 1999.

24. D. L. Pham and J. L. Prince, "Adaptive fuzzy segmentation of magnetic resonance images." IEEE Transactions on Medical Imaging, 18:737-752, 1999.

25. J. C. Rajapakse and F. Kruggel, "Segmentation of MR images with intensity inhomogeneities." Image and Vision Computing, 16:165-180, 1998.

26. E. A. Vokurka, N. A. Thacker, and A. Jackson, "A fast model independent method for automatic correction of intensity nonuniformity in MRI data." Journal of Magnetic Resonance Imaging, 10:550-562, 1999.

27. S. H. Lai and M. Fang, "A new variational shape-from-orientation approach to correcting intensity inhomogeneities in magnetic resonance images." Medical Image Analysis, 3:409424, 1999.

28. R. P. Velthuizen, J. J. Heine, A. B. Cantor, H. Lin, L. M. Fletcher, and L. P. Clarke, "Review and evaluation of MRI nonuniformity corrections for brain tumor response measurements." Medical Physics, 25:1655-1666, 1998.

29. B. Likar, J. B. A. Maintz, M. A. Viergever, and F. Pernuš, "Retrospective shading correction based on entropy minimization." Journal of Microscopy, 197:285-295, 2000.

30. W. H. Press, B. P. Flannery, S. A. Teukolosky, and W.T. Vetterling, "Numerical Recepies in C." Second Edition, Cambridge University Press, 1992.

31. C. A. Cocosco, V. Kollokian, R. K.-S. Kwan, and A. C. Evans, "BrainWeb: Online Interface to a 3D MRI Simulated Brain Database." NeuroImage 5:S425, 1997. Available: http://www.bic.mni.mcgill.ca/brainweb/

32. R. K.-S. Kwan, A. C. Evans, and G. B. Pike, "An Extensible MRI Simulator for PostProcessing Evaluation." in Visualisation in Biomedical Computing, Proceedings of VBC'96, Lecture Notes in Computer Science, 1131:135-140, 1996, Springer-Verlag.

33. D. L. Collins, A. P. Zijdenbos, V. Kollokian, J. G. Sled, N. J. Kabani, C. J. Holmes, and A. C. Evans, "Design and Construction of a Realistic Digital Brain Phantom." IEEE Transactions on Medical Imaging, 17:463-468, 1998.

34. ftp://ftp.bic.mni.mcgill.ca/pub/mni_n3/

35. http://www.niac.man.ac.uk/

36. R. P. Velthuizen, L. P. Clarke, S. Phuphanich, L. O. Hall, A. M. Bensaid, J. A. Arrington, H. M. Greenberg, and M. L. Silbiger. "Unsupervised measurement of brain tumor volume on MR images.” Journal of Magnetic Resonance Imaging 5:594-605, 1995.

37. J. Talairach and P. Tournoux, "Co-planar stereotaxic atlas of the human brain." New York: Thieme, 1988.

38. P. A. Filipek, D. N. Kennedy, and V. S. Caviness, "Volumetric analysis of central nervous system neoplasm based on MRI." Pediatric Neurology, 7:347-351, 1991.

39. D. N. Kennedy, P. A. Filipek, and V. S. Caviness, "Anatomic segmentation and volumetric calculations in nuclear magnetic resonance imaging." IEEE Transactions on Medical Imaging, 8:1-7, 1989. 